\title{
Randomised trial of corticosteroids in the treatment of tuberculous pleurisy
}

\author{
I Galarza, C Cañete, A Granados, R Estopà, F Manresa
}

\begin{abstract}
Background - Tuberculous pleurisy can result in pleural fibrosis, calcification and thickening. To prevent these complications, corticosteroids are frequently used in addition to antituberculous drugs; however, new therapeutic regimens can control the disease and minimise the sequelae, and there is no convincing evidence of the benefit of the use of corticosteroids as adjuvant therapy.

Methods - Patients received isoniazid $5 \mathrm{mg} / \mathrm{kg}$ and rifampicin $10 \mathrm{mg} / \mathrm{kg}$ daily for six months. Additionally, they were randomly assigned to a double blind treatment with either prednisone $(1 \mathrm{mg} / \mathrm{kg} / \mathrm{day}$ for 15 days and then tapering off) or placebo during the first month of treatment. Different clinical, radiological, and functional parameters were evaluated to assess the effect of corticosteroids.

Results - Fifty seven patients received prednisone and 60 placebo. At the end of the treatment the clinical outcome, the rate of reabsorption of the pleural fluid, the pleural sequelae, as well as lung capacity were similar in both groups.

Conclusions - Corticosteroids do not influence the clinical outcome or the development of long term pleural sequelae in tuberculous pleurisy.

(Thorax 1995;50:1305-1307)
\end{abstract}

Keywords: tuberculosis, pleural effusion, corticosteroids.

Pleural involvement is one of the most frequent extrapulmonary manifestations of tuberculosis. ${ }^{1}$ Organisation and fibrosis of the pleural exudate can result in long term pleural complications such as thickening, calcification, or fibrosis, causing restrictive ventilatory defects.

The treatment of tuberculous pleurisy includes the use of corticosteroids as adjuvant therapy to provide a prompt relief of the symptoms, to hasten absorption of fluid, and to prevent fibrosis and adhesions. Most information about adjuvant use of corticosteroids is based on uncontrolled studies and in trials using non-bactericidal drugs. ${ }^{23}$ Nowadays, even though the use of corticosteroids is still being recommended, ${ }^{4-6}$ there is no convincing evidence of the advantage of their use in the treatment of this disease.

For the treatment of tuberculous pleurisy the same regimen as for pulmonary tuberculosis is usually recommended. However, Dutt et al have demonstrated the efficacy of a six month regimen containing rifampicin and isoniazid for smear negative, culture positive pulmonary tuberculosis, ${ }^{7}$ and the same authors have recently reported on the efficacy of this two drug regimen for pleural tuberculosis. ${ }^{8}$

We present a prospective, randomised, double blind, placebo controlled study evaluating the role of corticosteroids in the control of fever and in the prevention of long term pleural complications of tuberculous pleurisy.

\section{Methods}

All patients admitted to the hospital for a pleural effusion of tuberculous aetiology between January 1985 and December 1992 were entered into the study. A definite microbiological or pathological diagnosis was obtained in $63 \%$ of all the patients. Patients with HIV infection were excluded. Further clinical and epidemiological data on the patients are shown in the table. Patients received isoniazid, $5 \mathrm{mg} / \mathrm{kg} /$ day or a total daily dose of $300 \mathrm{mg}$, and rifampicin, $10 \mathrm{mg} / \mathrm{kg} /$ day or a total daily dose of $600 \mathrm{mg} /$ day, once a day for six months as a combination tablet.

Additionally, patients were randomly assigned to receive, in a double blind fashion, either prednisone (corticosteroid group) or placebo (placebo group). Prednisone was administered in a single oral dose of $1 \mathrm{mg} / \mathrm{kg} /$ day during the first 15 days, and then gradually tapered off as follows: to $0.5 \mathrm{mg} / \mathrm{kg} /$ day from day $16-20$ of treatment, then to $0.25 \mathrm{mg} / \mathrm{kg} /$ day from day $21-26$, and finally to $0 \cdot 10 \mathrm{mg} / \mathrm{kg} /$ day for the remaining days of the month. The total period of corticosteroid (or placebo) treatment was 30 days.

Chest radiography, standard spirometric tests (forced vital capacity (FVC), forced expiratory volume in one second $\left(\mathrm{FEV}_{1}\right)$, and $\%$ FEV $_{1}$ ) (Vicatest VCT-4, Mijnhardt BV, Holland), blood biochemistry, and complete blood count were performed on all the patients at the entry into the study before the introduction of treatment. Our procedure for the routine laboratory analysis of pleural fluid in tuberculous pleurisy has been previously reported. ${ }^{9}$

Before the patient was discharged, pleural fluid was drained until a third of the hemithorax was observed to be occupied in a standard chest radiograph. On discharge the patients were regularly seen in the outpatient clinic to monitor the course of the disease, drug compliance, and possible side effects. Chest radiography and standard spirometric tests were repeated at months $1,2,4,6$, and 12 . Analytical assessments were performed at months 1,2 , and 6 . 
Characteristics of the study population

\begin{tabular}{llll}
\hline & $\begin{array}{l}\text { Corticosteroids } \\
\text { group }(n=57)\end{array}$ & $\begin{array}{l}\text { Placebo } \\
\text { group }(n=60)\end{array}$ & \\
\hline Age (years) & $26(11-53)$ & $28(14-53)$ & NS \\
M:F & $33: 27$ & $30: 31$ & NS \\
Side & & 36 & NS \\
$\quad$ Right & 34 & 24 & NS \\
Left & 23 & $4 \cdot 15(0-60)$ & NS \\
Fever (days) & $3 \cdot 32(0-50)$ & $2 \cdot 23(0-15)$ & NS \\
Thickening (mm) & $1 \cdot 77(0-40)$ & $95(63-140)$ & NS \\
FVC (\% predicted) & $95(65-130)$ & $46(12-96)$ & \\
Follow up (months) & $46(12-94)$ & & \\
\hline
\end{tabular}

Values are mean (range). NS $=$ no significant differences $(p>0.05)$.
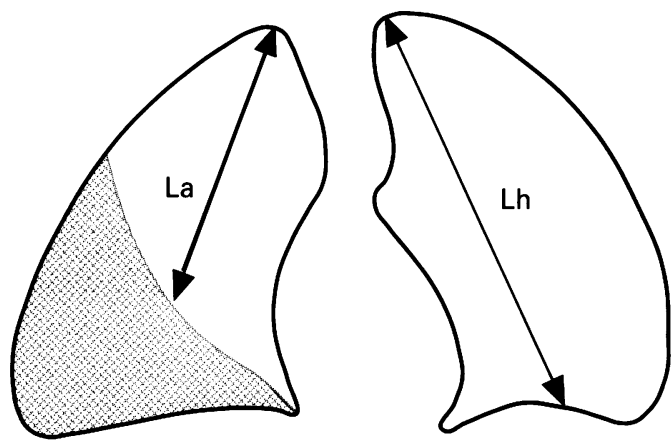

Figure 1 Reabsorption of pleural fluid was measured on the chest radiograph as a ratio of the length of the affected hemithorax (La) in relation to the healthy one (Lh). Index of reabsorption $=($ La $/ \mathrm{Lh}) \times 100$.

The effects of corticosteroids were evaluated by (1) days of fever after introduction of the treatment; (2) FVC at the end of the treatment; (3) maximal pleural thickening which was measured in millimetres at the costal margin of the affected hemithorax in a posteroanterior chest radiograph before and at months 1,6 , and 12; and (4) rate of reabsorption which was obtained as follows. A line was drawn from the apex of the lung to the half point in the curve of the liquid profile on the affected side (La) and to the mid point of the hemidiaphragmatic curve of the contralateral hemithorax (Lh), as shown in fig 1 . La was divided by $\mathrm{Lh}$ and expressed as a percentage. This index reflects the amount of liquid remaining in the pleural

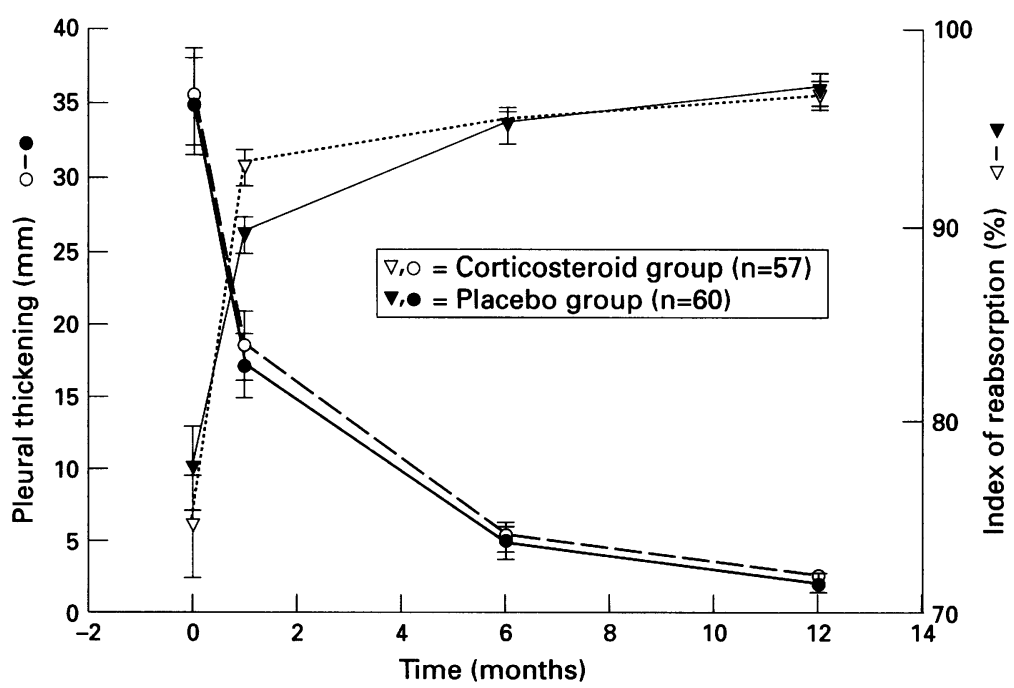

Figure 2 Comparative course of the index of reabsorption (\%) and pleural thickening $(\mathrm{mm})$ with treatment and thereafter in the two groups of patients. Results expressed as mean (SE). cavity and its evolution in time indicates the degree of reabsorption of the effusion.

\section{DATA ANALYSIS}

Statistical comparisons between the two groups were made by means of analysis of variance for repeated measures and the unpaired Student's $t$ test.

\section{Results}

Of the 117 patients entered into the study, 57 received prednisone and 60 placebo. Both groups were comparable in terms of age, sex, duration of the disease, distribution of symptoms, and time of follow up (table).

The duration of fever during treatment was similar in both groups: a mean duration of 3.32 (range 0-50) days for the corticosteroid group and $4.15(0-60)$ days for the placebo group $(p>0.05)$. At the end of the treatment period FVC was also similar in both groups (mean FVC 95\% (65-130\%) for the steroid group and $95 \%(63-140 \%)$ for the placebo group $(\mathrm{p}>0.05))$.

Data on pleural changes are shown in fig 2. The only slight but significant difference between the two groups was the reabsorption index measured at month 1 of treatment: $89 \%$ $(76-113 \%)$ in the placebo group compared with $93 \%(70-119 \%)$ in the other group ( $p=$ $0 \cdot 01)$. At the end of the treatment period only one patient in the steroid group $(40 \mathrm{~mm})$ and five in the placebo group ( $15 \mathrm{~mm}$ in three cases and $10 \mathrm{~mm}$ in the other two) showed some pleural thickening; these differences were not significant.

No side effects due to corticosteroids were observed in the 57 patients treated with prednisone.

\section{Discussion}

The use of the correct combination of bactericidal drugs ensures a rapid and more effective control of tuberculosis. For pleural effusions this results in better control of the clinical manifestations and inflammatory reaction, with a lower rate of pleural complications.

Lee et al performed a prospective placebo controlled study in 40 patients and showed the positive effect of the additional use of corticosteroids in this disease, particularly in terms of a more rapid resolution of fever. ${ }^{10}$ We have also observed a rapid decline in fever in the group on prednisone, but the difference was not significant. This difference between the two studies could be explained by the size of the sample and/or the different total dose of corticosteroids used. More recently, just a few months before the conclusion of our study, Dutt et al reported on the results of the same six month regimen for the treatment of tuberculous pleurisy with good results. ${ }^{8}$

We have also observed a significantly more rapid reabsorption of the effusion one month after the introduction of the treatment with prednisone, but this difference disappears thereafter (fig 2). Given the natural different 
length of the hemithoraces, an inaccurate result could be expected if the distribution of localisation in both groups was unbalanced. The similar distribution of right (34 in the corticosteroid group and 36 in the placebo group) and left (23 in the corticosteroid group and 24 in the placebo group) effusions in both groups avoided a significant bias. The possible confounding effect of localisation was therefore accounted for by using a two way repeated measures analysis of variance that showed no significant interaction between treatment and localisation, and a significant effect of corticosteroids after one month of treatment $(F=$ $6 \cdot 82 ; \mathrm{p}=0 \cdot 01$ ).

Although one patient in the corticosteroid group and five in the placebo group showed some pleural thickening at the end of the study, no significant difference was observed between the two groups (fig 2). As expected from the clinical and radiological signs seen at six months, no significant changes in FVC were observed at the end of the treatment.

Reducing the volume of pleural fluid to a third of a hemithorax could explain the absence of pleural sequelae in both groups. Repeated drainage of the pleural effusion was a common procedure to avoid pleural complications until Grewal et $a l^{11}$ reported better results with corticosteroids; however, this positive effect was obtained before the routine use of rifampicin and isoniazid, and better therapeutic regimens were introduced. Our only interest in draining large amounts of fluid was to equalise the volume of liquid to be reabsorbed as a result of antituberculous therapy.

It can be argued that a complete drainage of the hemithorax associated with the use of a drug regimen using pyrazinamide would give better results, but if we consider that tuberculous pleurisy is a disease caused by a small number of bacilli, and that pyrazinamide is bactericidal particularly for slow growing and for intracellular organisms, the clinical relevance of its addition to a regimen containing two good bactericidal drugs is yet to be proven.

Rifampicin diminishes the anti-inflammatory effects of prednisone ${ }^{12}$ so that the precise dos- age of prednisone cannot be stated in the therapeutic regimen we have used. In fact, the slight differences observed between the two groups may be related, at least in part, to this pharmacological interaction. It could also be argued that by using a larger dose (daily or total) of prednisone, a definite favourable effect could be achieved, but our results in the placebo group support the view that further studies using a higher dose of prednisone are not recommended.

We can conclude that the advantage of adding corticosteroids to an antituberculous regimen is not clinically relevant, and that its routine use in the treatment of tuberculous pleurisy is not indicated.

The authors acknowledge a grant from Fondo de Investigaciones de la Seguridad Social (FISS $n^{\circ}$ 87/0986) and Accesit SEPAR 1987.

1 Seibert AF, Haynes J Jr, Middleton R, Bass JB Jr. Tuberculous pleural effusion. Twenty years experience. Chest 1991;99:883-6.

2 Aspin J, O'Hara H. Steroid-treated tuberculous pleural effusions. Br F Tuberc Dis Chest 1958;52:81-3.

Menon NK. Steroid therapy in tuberculous effusion. Tubercle 1964;45:17-20.

4 Senderovitz T, Viskum K. Corticosteroids and tuberculosis. Respir Med 1994;88:561-5.

5 American Thoracic Society. Treatment of tuberculosis and tuberculosis infections in adults and children. Am Rev Respir Dis 1986;134:355-63.

6 Seaton A, Seaton D, Leitch AG. Crofton and Douglas's Respiratory Diseases. 4th ed. Oxford: Backwell Scientific Publications, 1989:435.

7 Dutt AK, Moers D, Stead WW. Smear negative, culture positive pulmonary tuberculosis. Six months chemotherapy with isoniazid and rifampin. Am Rev Respir Dis therapy with isoniazi

8 Dutt AK, Moers D, Stead WW. Tuberculous pleural effusion: six months therapy with isoniazid and rifampin. $A m$ Rev Respir Dis 1992;145:1429-32.

9 Cañete C, Galarza I, Granados A, Farrero E, Estopá R, Manresa F. Tuberculous pleural effusion. Six month treatment with isoniazid and rifampicin. Thorax 1994;49:11601.

10 Lee $\mathrm{ChH}$, Wang WJ, Lan RS, Tsai YH, Chiang YCh. Corticosteroids in the treatment of tuberculous pleurisy. A double blind, placebo-controlled, randomized study. Chest 1988; 84:1256-9.

11 Grewal KS, Dixit RP, Sil DR. A comparative study of therapeutic regimens with and without corticosteroids in the treatment of tuberculous pleural effusion. $\mathcal{F}$ Indian Med Ass 1969;52:514-6.

12 MacAllister WA, Thompson PJ, Al-Habet SM, Rogers HJ Rifampicin reduces effectiveness and bioavailability of prednisone. BMf 1983;286:923-5. 